\title{
Cold pressor pain assessment based on EEG power spectrum
}

\author{
$\mathrm{Li} \mathrm{Wang}^{1} \cdot$ Yan Xiao $^{2} \cdot$ Richard D. Urman $^{3} \cdot$ Yingzi Lin $^{1}$ (D)
}

Received: 4 April 2020 / Accepted: 1 November 2020 / Published online: 10 November 2020

(c) Springer Nature Switzerland AG 2020

\begin{abstract}
Patients' pain level of the patients is a fundamental factor for the patients' anesthetics usage. To investigate the responses of electroencephalogram (EEG) to cold pressor pain in the frequency domain, twenty healthy subjects' EEG signals were recorded during a cold pressor test. A band-pass filter and independent component analysis were used to preprocess the EEG signal. Fast Fourier transformation as used to analyze the EEG power spectrum. Paired t-tests were performed on the EEG power to investigate the significance of the difference between pain and no-pain EEG power. In pain state, the EEG power increased significantly at $\mathrm{EEG}$ channel location $\mathrm{Cz}, \mathrm{O} 1$, and $\mathrm{O} 2$ over most frequency bands: 1-4 Hz, 4-8 Hz, 8-13 $\mathrm{Hz}, 13-30 \mathrm{~Hz}, 30-50 \mathrm{~Hz}$. The EEG power of the gamma band increased over extensive brain regions in pain condition. This study suggests that the power of EEG data in pain state has a higher value over most frequency bands. The central and occipital brain regions show the most significant differences between no-pain and pain state. The findings of this study provide evidence that EEG is a promising tool to objectively assess the pain level of human beings.
\end{abstract}

Keywords Objective pain assessment · EEG · Power spectrum · Cold pressor test

\section{Introduction}

Patients' pain level is a key factor to decide the patients' usage of anesthetics in clinical practice. Getting an objective pain level of patients is crucial for both patients and doctors. At the moment, most hospitals are using the numerical rating scale (NRS) [1, 2], verbal rating scale (VRS), visual analog scale (VAS) [3, 4], and faces scale [5], as shown in Fig. 1. The NRS asks the patients to report their pain level on a scale from 0 to 10, with the description that 0 means "no pain" and 10 means "excruciating pain". The VRS provides a list of adjectives with the meaning of different pain levels.

The patients will select one adjective to describe their pain level. The VAS usually shows patients a $10 \mathrm{~cm}$ line, on which one end is "no pain" and the other end is "excruciating pain". The patients will point a location on the line that best represents their pain level [6]. The faces scale is usually comprised of a few facial expressions, indicating the pain level. These methods are simple and easy to use. However, biased pain ratings could be reported for a few reasons. First, these methods are subjective, which means the reported pain level is totally based on that patient's personal opinion; Second, these methods fail to work when patients are unconscious; Third, some patients could lie on their pain level to cheat on unwarranted absence from work or prescription of opioid [7], which caused an enormous economic burden for the nation. In a study on the economic burden, it's estimated that the total economic burden of prescription opioid overdose, abuse, and dependence was $\$ 78.5$ billion in the United States in the year 2013 [8].

Cold pressor test (CPT) was first introduced by Hines Jr and Brown [9]. The key step of CPT is to place one of the subject's hands into cold water up to the wrist. Wolf and Hardy's work proved that pain could be conveniently

$\triangle$ Yingzi Lin, yi.lin@northeastern.edu | 'Intelligent Human-Machine Systems Laboratory, Northeastern University, Boston, MA 02115, USA. ${ }^{2}$ University of Texas at Arlington, Arlington, TX 76019, USA. ${ }^{3}$ Brigham and Women's Hospital, Harvard Medical School, Boston, MA 02115, USA. 


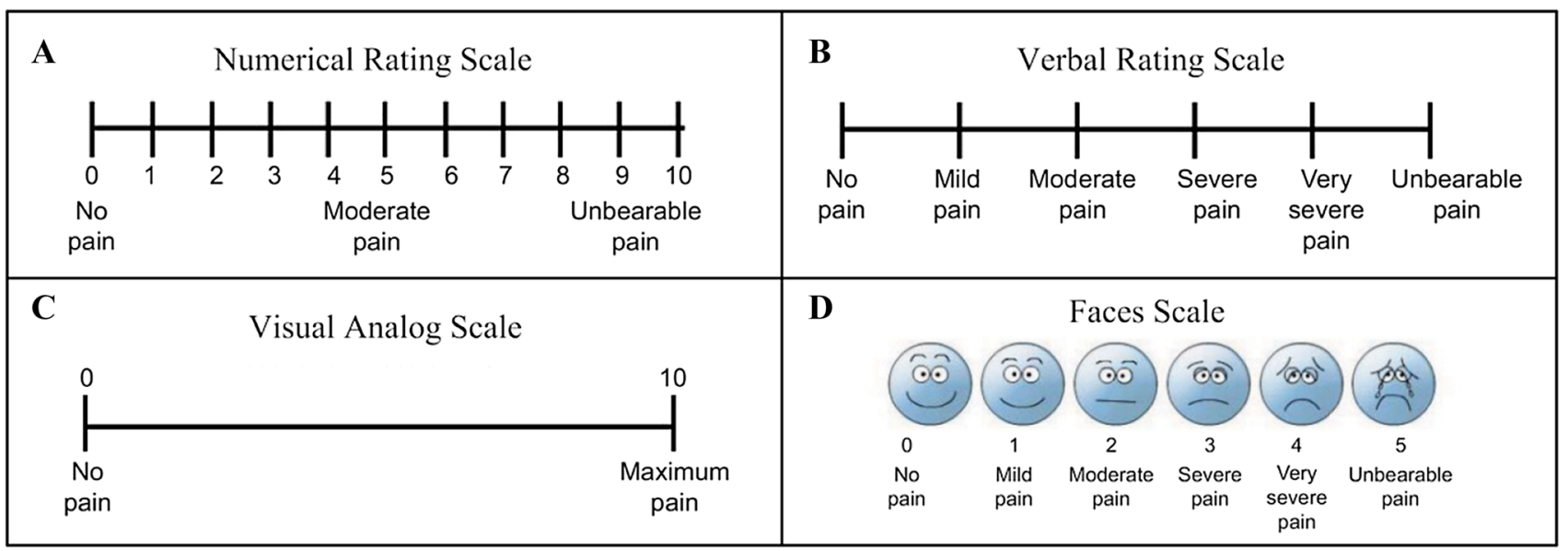

Fig. 1 A few examples of common pain rating scales used in most hospitals. a numerical rating scale (NRS), b verbal rating scale (VRS), c visual analog scale (VAS), $\mathbf{d}$ faces scale [5]

produced using CPT and its intensity depended directly on the degree of cooling [10]. The CPT has been commonly used as an experimental tonic pain model in the past decades. Recently, Hansen et al. [11] investigated the characterization of cortical source generators using CPT. Barati et al. [12] conducted an experiment on hemodynamic response using CPT as a pain stimulus.

Electroencephalogram (EEG) is the electrical activity of the human brain, and has been extensively studied in different scenarios $[13,14]$. As a powerful signal to study the electrophysiological dynamics of the human brain, EEG has been believed to be a pain indicator. Prior research showed that the EEG signal was a promising indicator to assess pain level. Jensen et al. [3] conducted a study, showing that certain EEG patterns were related to chronic pain in people with spinal cord injury. In the study of Diers et al. [15], some components of the EEG signal between healthy subjects and chronic low back pain patients (CLBP) were significantly different. However, the results of different studies varied greatly. It's found that, during the cold pain condition, alpha band $(8-12 \mathrm{~Hz})$ power increased over the posterior scalp and decreased over the contralateral temporal scalp. Gamma band $(25-100 \mathrm{~Hz})$ power increased over most of the scalp regions [16]. According to Shao et al. [17], the band power $(8-12 \mathrm{~Hz})$ of the EEG signal decreased and the band power $(18-30 \mathrm{~Hz})$ increased over extensive brain regions in cold pain condition.

The present study aims to assess people's pain level using the EEG power spectrum. Towards this aim, 20 subjects were recruited for the CPT. A band-pass filter and independent component analysis (ICA) were performed for signal preprocessing. A fast Fourier transform (FFT) was used to analyze the EEG signal power spectrum. The differences between pain and no-pain states were also examined in topographies over different frequency bands. The remaining parts of this paper are structured as follows: Sect. 2 represents the methodology of this study. Sect. 3 shows the experimental results. In Sect. 4, we present the discussion and limitation of this research. Sect. 5 is the conclusion of the research.

\section{Materials and methods}

\subsection{Subjects}

Twenty healthy young adults ( 17 males and 3 females, age: mean $=27.4, S D=5.7$ ) participated in this experiment. None of them had any history of neurological, psychiatric, and cardiovascular problems. None of the subjects experienced any sort of pain before participating in the experiment. They were instructed to avoid alcohol and caffeine in the 24 hours before the experiment because alcohol and caffeine affect pain perception $[18,19]$. Each subject was given a detailed explanation of the experimental procedures and signed a consent form upon his/ her arrival. All experiments were conducted in Intelligent Human-Machine Systems Lab at Northeastern University (NEU). This research was approved by the NEU Institutional Review Board (IRB\#:17-01-25).

\subsection{Apparatus}

(1) Enobio EEG device (Neuroelectrics, Barcelona, Spain) was used to record EEG in this study. The sampling rate of our EEG device was $500 \mathrm{~Hz}$. Enobio transferred EEG data to a computer through Wi-Fi. (2) Ice water $\left(\sim 0{ }^{\circ} \mathrm{C}\right)$ was used as pain stimulation in this study. (3) A Dell workstation operating on windows 7 with 16 G RAM was used to process the data in this study. 


\subsection{Experimental procedure}

Each subject signed a consent form before the experiment. During the experiment, the subjects were asked to sit in a comfortable armchair, with a monitor facing them at a distance of about $30 \mathrm{~cm}$. The experiment took place in a quiet room without any disturbances. The subjects were asked to look at a green dot on a monitor in front of them and keep their eyes open during the entire experiment. The experimental procedures were as follows: Step 1, EEG sensors were attached to the subjects based on the international 10-20 system [20]. Step 2 , the subjects were asked to rest in an armchair for 3 min before the experiment started. Step 3, baseline data recording. To reduce the subjects' eye movement and keep their eyes open during the experiment, they were asked to look at a green dot on the monitor (same in the following steps). The baseline data collection lasted 20 s. Step 4, the subjects were asked to put their right hands (up to the wrist) into a bucket filled with ice water. There was a letter " $P$ " showing up on the monitor every $20 \mathrm{~s}$, as shown in Fig. 2. The subjects were asked to report their pain level, using VRS, every time when they see the letter "P" on the screen. The subjects were asked to do this for a maximum period of $200 \mathrm{~s}$. If the subjects could not tolerate the pain for that amount of time, the subject could elect to withdraw their hands from the ice water, effectively ending that experimental session. Step 5 , the experiment ended.

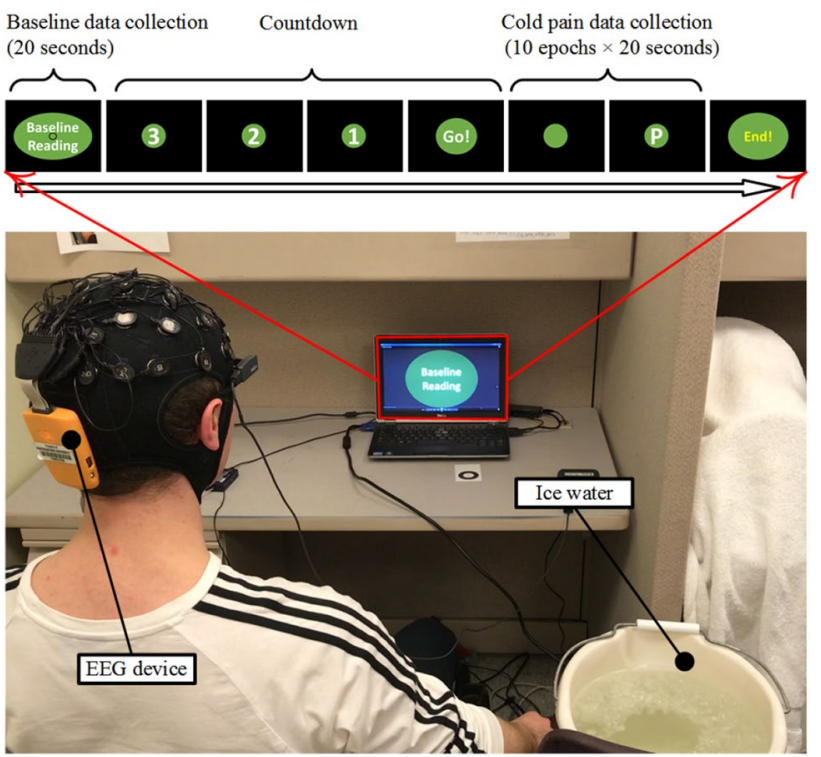

Fig. 2 Schematic diagram of the experimental setup (the upper part: the screen displays shown to the subjects; the lower part: the experimental scene)

\subsection{Sample size calculation}

The sample size calculation was performed in Minitab (Version 17, Minitab, PA, USA). Based on a previous similar study [21], the difference of EEG spectral power (relative value) in different pain states was around $4 \%$. With the desired power of 0.8 and a significance level of 0.05 , a sample size of 15 participants was generated for this study. Considering the variation of EEG power in different channels, we recruited 20 subjects in total to achieve the desired power.

\subsection{Data acquisition and preprocessing}

EEG signals were recorded in 11 locations (Fp1, Fp2, F7, $\mathrm{F} 8, \mathrm{~T} 7, \mathrm{~T} 8, \mathrm{C3}, \mathrm{C} 4, \mathrm{Cz}, \mathrm{O} 1, \mathrm{O} 2)$, as shown in Fig. 3. EEG data were collected with a sampling rate of $500 \mathrm{~Hz}$ and the impedances of electrodes were less than $5 \mathrm{~K} \Omega$. Different subjects had different lengths of EEG data because they were allowed to withdraw from the experiment at any time. In the present study, for an easier comparison, we chose to compare the baseline data with the pain data that had the highest subjective pain rating. The EEG data were filtered with a $1-50 \mathrm{~Hz}$ band-pass filter. An ICA was performed using ICA toolbox of EEGLAB (EEGLAB, SCCN [22]). We used ADJUST add-on [23] in EEGLAB to remove the artifacts in the raw EEG signal.

\subsection{EEG data analysis}

For power spectral analysis, FFT was performed to convert the EEG signal from the time domain to the frequency domain and calculate the power spectrum. In the present study, the target frequency bands were: delta (1-4 Hz), theta $(4-8 \mathrm{~Hz})$, alpha $(8-13 \mathrm{~Hz})$, beta $(13-30 \mathrm{~Hz})$, gamma $(30-50 \mathrm{~Hz})$. The power spectrum of frequency bands was calculated for each channel location and experimental condition (pain vs. no-pain). Paired t-tests are conducted

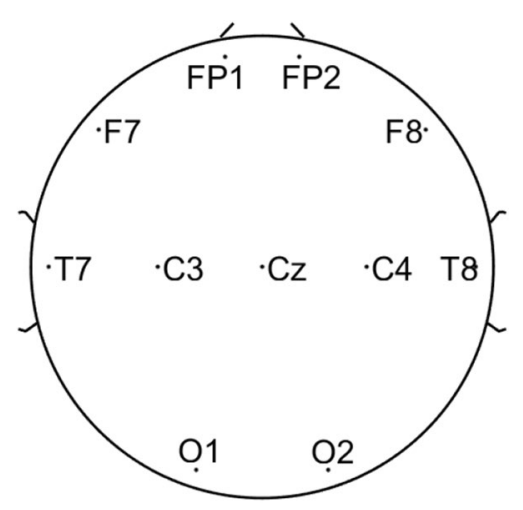

Fig. 3 EEG channel location map according to 10-20 system [20] 
on each channel location and frequency band to evaluate the significance level of differences between cold pain and no-pain condition.

\section{Results}

\subsection{Subjective pain level}

The average subjective pain ratings with standard deviation are shown in Fig. 4. According to the IRB protocol, our subjects could stop the experiment at any time. As noted in Fig. 4, the standard deviations of the pain ratings were zero at the end of the experiment. That's because only one subject made it to the end of the experiment. The baseline pain level for all subjects was zero since none of them reported any type of pain in the experimental survey before participating in the experiment. The pain level of the two chosen conditions (pain vs. no-pain) was significantly different (paired t-test, DOF $=19, p<0.01$ ). Some subjects' pain level increased with the experiment going on and decreased at the end of the experiment. It's pointed out that numbness could be a reason that the subjects' pain level decreased at the end of the cold pain experiment [24].

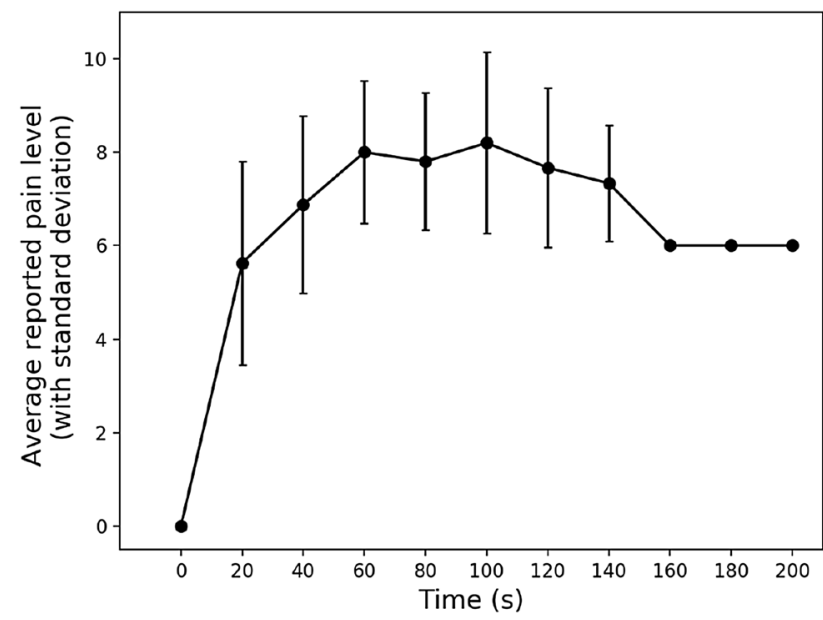

Fig. 4 The average pain level of all subjects at the end of each epoch with standard deviation

\subsection{EEG in frequency domain}

ICA and ADJUST were used to remove artifacts in the raw EEG data. Four kinds of artifacts were detected and removed (Fig. 5). As shown in Fig. 6, the left figure shows raw EEG data and the right figure shows EEG data filtered by ICA.

EEG power was calculated over five frequency bands (delta: 1-4 Hz, theta: 4-8 Hz, alpha: 8-13 Hz, beta: $13-30 \mathrm{~Hz}$, gamma: $30-50 \mathrm{~Hz}$ ) at each EEG channel location. The averaged EEG power of each frequency band is shown in topographies (Fig. 7). In general, the power of each frequency band increased over extensive brain regions. Paired t-tests were conducted to analyze the significance level of difference between pain and no-pain conditions at each channel. $p$-values of each channel from the paired t-tests are also interpreted as topographies in the last column of Fig. 7.

We compared the differences between the two conditions of each frequency band at each EEG channel location (paired t-test, DOF $=19$ ). The significance level is shown in Table 1. Delta band $(1-4 \mathrm{~Hz})$ showed significant differences at $\mathrm{Cz}(p<0.03, \mathrm{DOF}=19)$ and over the occipital region $(p$ $<0.03(\mathrm{O} 1), p<0.05(\mathrm{O} 2), \mathrm{DOF}=19)$. Theta band $(4-8 \mathrm{~Hz})$ showed significant differences at $\mathrm{C} 3(p<0.04, \mathrm{DOF}=19)$, $\mathrm{Cz}(p<0.03, \mathrm{DOF}=19)$ and, $\mathrm{O} 1(p<0.03, \mathrm{DOF}=19)$. Alpha band $(8-13 \mathrm{~Hz})$ showed significant differences at FP2 $(p<$ $0.03, \mathrm{DOF}=19), \mathrm{Cz}(p<0.03, \mathrm{DOF}=19)$, and over the occipital region $(p<0.02(01), p<0.05(\mathrm{O} 2), \mathrm{DOF}=19)$. Beta band $(13-30 \mathrm{~Hz})$ showed significant differences over frontal pole region $(p<0.03(\mathrm{FP} 1), p<0.03(\mathrm{FP} 2), \mathrm{DOF}=19)$, occipital region $(p<0.01(01), p<0.01(\mathrm{O} 2), \mathrm{DOF}=19)$ and central region $(p<0.01(\mathrm{Cz}), p<0.03(\mathrm{C} 3), \mathrm{DOF}=19)$. Gamma band $(30-50 \mathrm{~Hz}$ ) showed significant differences over extensive brain regions. In terms of brain regions, the central region and occipital region showed significant differences over almost all frequency bands.

\section{Discussion}

The present study explored the relationship between EEG power spectrum and pain level of CPT. We compared the EEG power spectrum in two different conditions (pain vs. no-pain). Specifically, the EEG power spectrum of gamma
Fig. 5 Four kinds of artifacts detected and removed by ADJUST [23]. a Horizontal eye movement, b Vertical eye movement, c Eye blinks, $\mathbf{d}$ Generic discontinuities (sudden amplitude fluctuations in a channel)

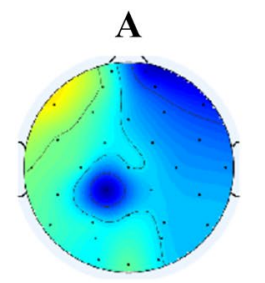

B

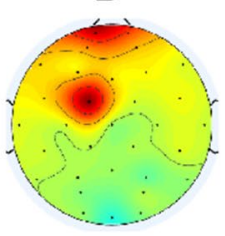

C

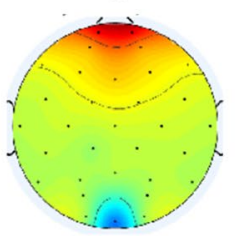

D

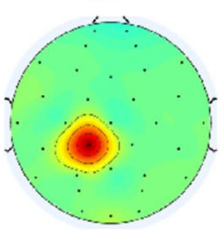

SN Applied Sciences 
A

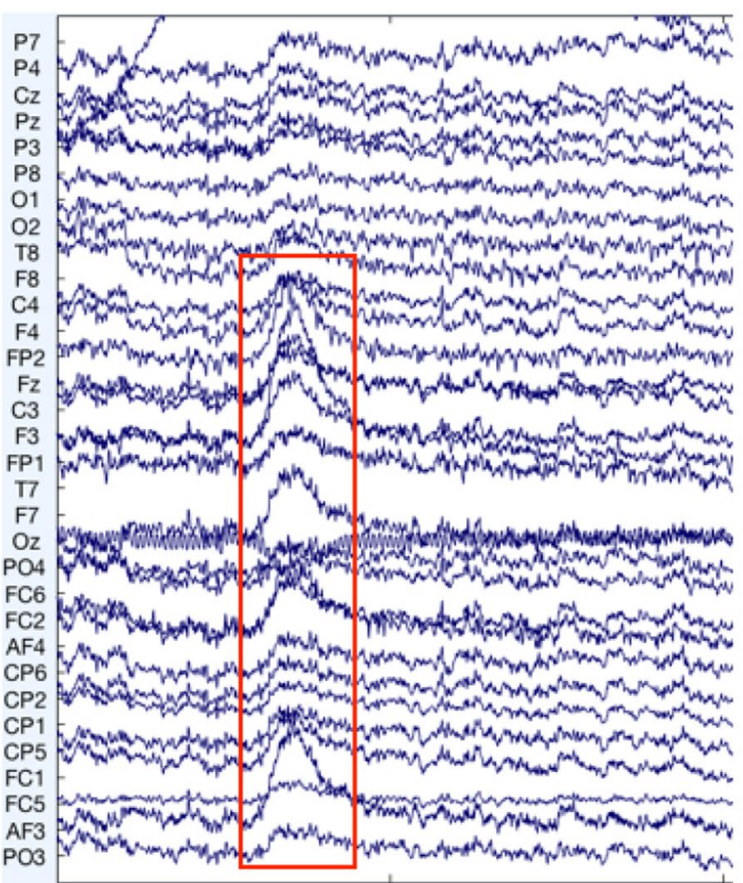

B

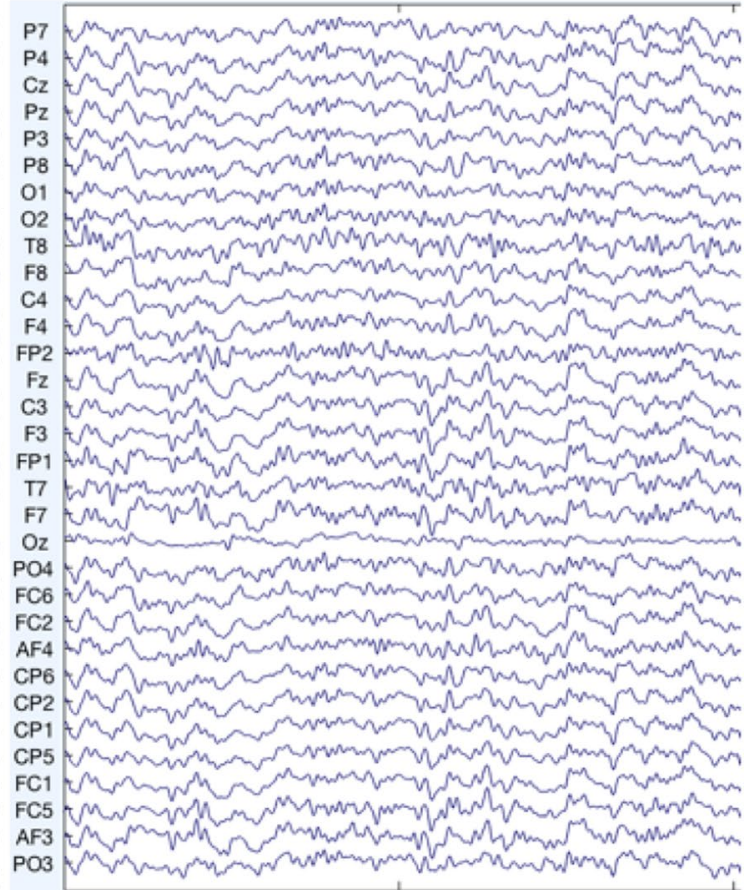

Fig. 6 a Raw EEG data before filtering (noise shown in the rectangle). b Filtered EEG data

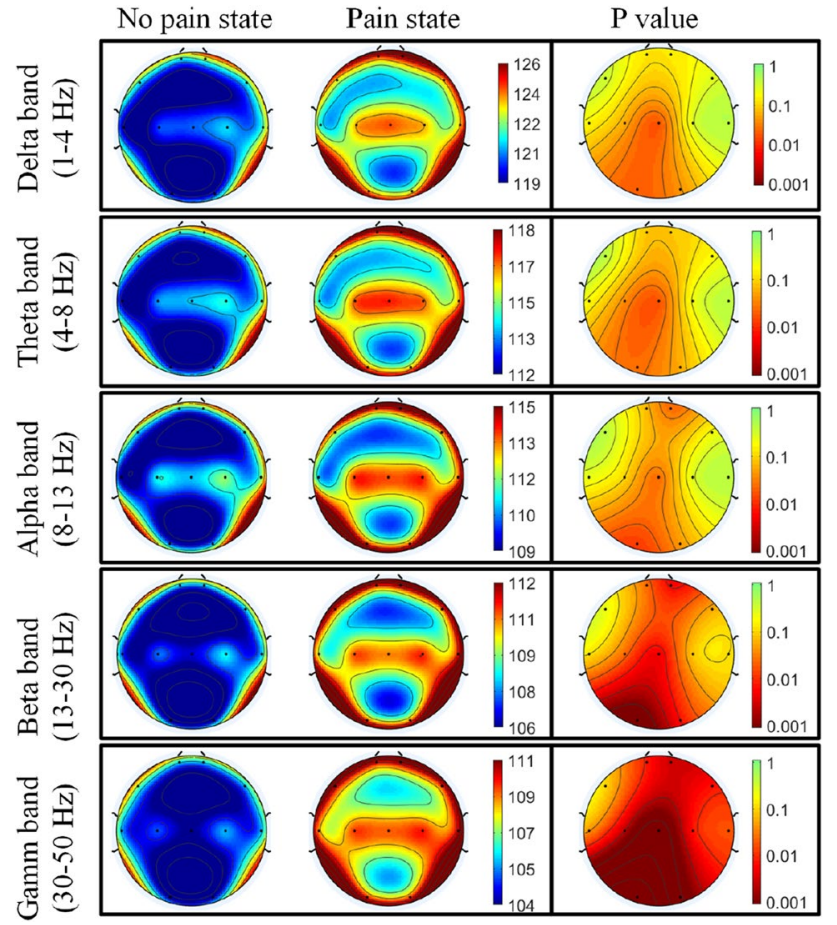

Fig. 7 EEG topographies comparing no-pain and pain states over five frequency bands. No pain state topographies (left most column). Pain state topographies (middle column). EEG power density unit: $10 * \log 10(\mathrm{uV} / \mathrm{Hz})$. The differences between pain and no pain states were tested using paired t-tests ( $p$-values shown as topographies (right most column), DOF $=19$ ) band increased significantly over extensive brain regions while the subjects were in the pain condition.

\subsection{Subjective pain rating}

In this study, we recruited 20 healthy subjects and used ice water to trigger pain. The amount of time that each subject could last in the ice water varied greatly. Two subjects lasted only 1 epoch (Each epoch was 20 s), while one subject made it to the end of the experiment (10 epochs). Figure 8 represents the distribution of total epochs that each subject had in the experiment (mean $=5, S D=3.18$ ). While in some other studies, all of their subjects made it to the end of the experiment $[16,17]$. The water temperature in their studies was controlled at a higher degree. The initial temperature of the cold water was $4.3 \pm 0.8^{\circ} \mathrm{C}$ in one study [16] and $10 \pm 1{ }^{\circ} \mathrm{C}$ in another study [17]. As mentioned previously, the subjects' hands got numb while staying in the ice water for a long time. In future studies, a higher temperature of the cold water is recommended in CPT.

\subsection{Power spectral analysis}

The EEG data were collected from 32 channels all over the scalp, although there was ample evidence showing that pain perception was related to the frontal [21,25], central [26], temporal [16], and parietal [16, 25] regions 
Table 1 Significance level of frequency bands at each location (pain vs. no-pain)

\begin{tabular}{|c|c|c|c|c|c|c|c|c|c|c|c|}
\hline \multirow[t]{3}{*}{ Frequency bands } & \multicolumn{11}{|c|}{ Channel locations } \\
\hline & \multicolumn{2}{|c|}{ Frontal pole } & \multicolumn{2}{|c|}{ Frontal } & \multicolumn{3}{|c|}{ Central } & \multicolumn{2}{|c|}{ Temporal } & \multicolumn{2}{|c|}{ Occipital } \\
\hline & FP1 & FP2 & F7 & F8 & $\mathrm{C} 3$ & $\mathrm{Cz}$ & $\mathrm{C} 4$ & T7 & T8 & $\mathrm{O} 1$ & $\mathrm{O} 2$ \\
\hline Delta (1-4 Hz) & - & - & - & - & - & 0.03 & - & - & - & 0.03 & 0.05 \\
\hline Theta $(4-8 \mathrm{~Hz})$ & - & - & - & - & 0.04 & 0.03 & - & - & - & 0.03 & - \\
\hline Alpha $(8-13 \mathrm{~Hz})$ & - & 0.03 & - & - & - & 0.03 & - & - & - & 0.02 & 0.05 \\
\hline Beta $(13-30 \mathrm{~Hz})$ & 0.03 & 0.01 & - & - & 0.03 & 0.01 & - & - & - & 0.01 & 0.01 \\
\hline Gamma (30-50 Hz) & 0.01 & 0.01 & - & 0.01 & 0.01 & 0.01 & 0.01 & 0.05 & 0.02 & 0.01 & 0.01 \\
\hline
\end{tabular}

- Means the difference is not significant

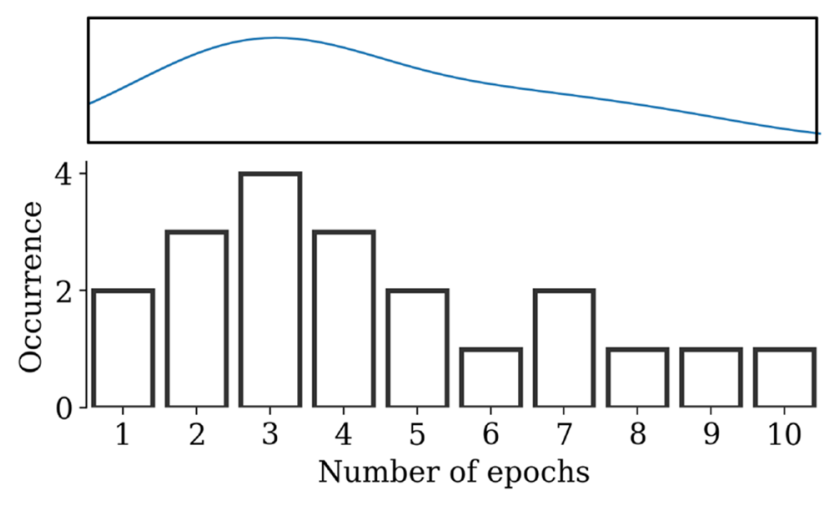

Fig. 8 The distribution of the total number of epochs each subject had in the experiment

on the scalp. The power spectral analysis of the EEG signals showed that the EEG power had a higher value over extensive brain regions in the pain condition. Moreover, the EEG power increased more significantly in higher frequency bands (beta and gamma). According to our result, delta band $(1-4 \mathrm{~Hz})$ showed significant increases in the EEG power at only three channel locations, while gamma band $(30-50 \mathrm{~Hz})$ showed significant increases over almost all measured brain regions.

We noted that there wasn't a commonly agreed conclusion of how the EEG band power changed based on the pain state. For faster brain activities (beta \& gamma), our results concurred with most CPT studies $[16,17,27]$, which stated that the faster EEG signals had greater power in pain condition. For slower brain activities (delta, theta, and alpha), our results were consistent with one study [25], while inconsistent with some other studies [16, 17]. It suggested that there was a significant and long-lasting increase of delta power in pain condition [25]. However, it showed that theta power decreased over left medial frontal and left superior frontal regions [17]. Of note, Dowman et al. [16] study illustrated that the change of alpha power varied depending on the EEG channel location. It demonstrated that alpha power decreased over the contralateral temporal region and increased over the posterior region in pain condition. According to a review study [28], stronger slow wave activities might indicate a low level of general mental ability. This implied subjects' mental ability was suppressed in the pain condition.

In the present study, the frontal and temporal regions showed hardly any significant differences, which contrasted with some studies, saying the frontal and temporal regions showed significant changes in EEG signals in different pain states $[16,21]$. The frontal lobe is usually involved in conscious thoughts, decision making, motor function, etc. The temporal lobe is usually associated with long-term memory, language comprehension, etc. According to a review of 6 CPT studies [16], three of the 6 studies reported EEG power changes over the frontal region. One of the 6 studies reported changes over the temporal region. The reason that there were hardly any significant differences over the frontal and temporal regions might be the artifacts in the signals and the usage of ICA. As is known, EEG signals in the frontal region can be easily contaminated by eye blinking. And ICA is a commonly used tool to get rid of artifacts from the EEG signal, but meanwhile, it may also remove some useful components in the raw signal [29].

\subsection{Limitation}

However, some limitations of this research should be noted. First, the data length of each subject was different, since the subjects could stop the experiment at any time. The differences of data lengths made it impossible to compare all data we collected. As a result, we used two trials from each subject (baseline trial and trial with the highest pain rating). In the future, a higher temperature of cold water is recommended for CPT. Second, we had 20 subjects with 17 males and 3 females in this study. The number of male subjects was much more than that of female subjects. In the future study, it is suggested to have more subjects and an equal number of male and female subjects. Further analysis of potential gender differences will be conducted for the cold pressure test. 


\section{Conclusions}

In this study, we aimed to develop a method to assess people's pain level objectively. We used ice water as a painful stimulus to healthy subjects and recorded subjects' EEG data. We applied ICA to EEG data to remove artifacts and used FFT to analyze EEG power spectrum. Paired t-tests were conducted to determine if there was a significant difference between the two experimental conditions. It's found that the EEG power spectrum, in general, had a higher value while in pain condition. The gamma band power showed significant differences over extensive brain regions. The EEG power spectrum is proved to be a promising and potential tool to objectively assess pain level of patients.

Acknowledgements The authors would like to thank the Capstone students for helping collect the EEG data and the subjects for participating in the experiment. The authors would like to thank NSF for supporting this project.

Author contributions Conceptualization: YL and YX; Methodology: YL and LW; Software: LW and YL; Validation: LW and YL; Formal analysis: LW and YL; Investigation: YL, YX and RDU; Resources: YL, YX and RDU; Data curation: LW and YL; Writing-original draft preparation: LW and YL; Writing-review and editing: YL, YX and RDU; Visualization: LW and YL; Supervision: YL, YX and RDU; Project administration: YL; Funding acquisition: $\mathrm{YL}, \mathrm{YX}$ and $\mathrm{RDU}$.

Funding This research has been financially supported by a collaborative National Science Foundation (NSF) project entitled Novel Computational Methods for Continuous Objective Multimodal Pain Assessment Sensing System (COMPASS) under the Award \#1838796, 1838650 and 1838621.

\section{Compliance with ethical standards}

Conflict of interest The authors declare that they have no conflict of interest.

\section{References}

1. Hartrick CT, Kovan JP, Shapiro S (2003) The numeric rating scale for clinical pain measurement: a ratio measure? Pain Pract 3(4):310-316

2. Childs JD, Piva SR (2005) Responsiveness of the numeric pain rating scale in patients with low back pain. Spine 30(11):1331-1334

3. Jensen MP, Chen C, Brugger AM (2003) Interpretation of visual analog scale ratings and change scores: a reanalysis of two clinical trials of postoperative pain. J Pain 4(7):407-414

4. Sriwatanakul K, Kelvie W, Lasagna L, Calimlim JF, Weis OF, Mehta $G$ (1983) Studies with different types of visual analog scales for measurement of pain. Clin Pharmacol Ther 34(2):234-239

5. Zubrzycki M, Liebold A, Skrabal C, Reinelt H, Ziegler M, Perdas E, Zubrzycka M (2018) Assessment and pathophysiology of pain in cardiac surgery. J Pain Res 11:1599
6. Turk DC, Melzack R (2011) Handbook of pain assessment. Guilford Press, New York

7. Levy N, Sturgess J, Mills P (2018) "Pain as the fifth vital sign" and dependence on the "numerical pain scale" is being abandoned in the us: Why? Br J Anaesth 120(3):435-438

8. Florence C, Luo F, Likang X, Zhou C (2016) The economic burden of prescription opioid overdose, abuse and dependence in the United States, 2013. Med Care 54(10):901

9. Brown GE, Hines EA Jr (1936) The cold pressor test for measuring the reactibility of the blood pressure: data concerning 571 normal and hypertensive subjects. Am Heart J 11(1):1-9

10. Wolf S, Hardy JD (1941) Studies on pain. Observations on pain due to local cooling and on factors involved in the "cold pressor" effect. J Clin Investig 20(5):521-533

11. Hansen TM, Mark EB, Olesen SS, Gram M, Frøkjær JB, Drewes AM (2017) Characterization of cortical source generators based on electroencephalography during tonic pain. J Pain Res 10:1401

12. Barati Z, Zakeri I, Pourrezaei K (2017) Functional near-infrared spectroscopy study on tonic pain activation by cold pressor test. Neurophotonics 4(1):015004

13. Lin Y, Wang L, Xiao Y, Urman RD, Dutton R, Ramsay M (2018) Objective pain measurement based on physiological signals. In: Proceedings of the International Symposium on Human Factors and Ergonomics in Health Care, vol 7, pp 240-247. SAGE, Los Angeles

14. Taheri S, Ezoji M, Sakhaei SM (2020) Convolutional neural network based features for motor imagery EEG signals classification in brain-computer interface system. SN Appl Sci 2(4):1-12

15. Diers M, Koeppe C, Diesch E, Stolle AM, Hölzl R, Schiltenwolf $M$, van Ackern K, Flor H (2007) Central processing of acute muscle pain in chronic low back pain patients: an EEG mapping study. J. Clin Neurophysiol 24(1):76-83

16. Dowman R, Rissacher D, Schuckers S (2008) EEG indices of tonic pain-related activity in the somatosensory cortices. Clin Neurophysiol 119(5):1201-1212

17. Shao S, Shen K, Ke Yu, Wilder-Smith EPV, Li X (2012) Frequencydomain EEG source analysis for acute tonic cold pain perception. Clin Neurophysiol 123(10):2042-2049

18. Horn-Hofmann C, Büscher P, Lautenbacher S, Wolstein J (2015) The effect of nonrecurring alcohol administration on pain perception in humans: a systematic review. J Pain Res 8:175

19. Tarnopolsky MA (2008) Effect of caffeine on the neuromuscular system-potential as an ergogenic aid. Appl Physiol Nutr Metabol 33(6):1284-1289

20. Jasper HH (1958) The ten-twenty electrode system of the international federation. Electroencephalogr Clin Neurophysiol 10:370-375

21. Huber MT, Bartling J, Pachur D, Woikowsky-Biedau S (2006) EEG responses to tonic heat pain. Exp Brain Res 173(1):14-24

22. Delorme A, Makeig S (2004) Eeglab: an open source toolbox for analysis of single-trial EEG dynamics including independent component analysis. J Neurosci Methods 134(1):9-21

23. Mognon A, Jovicich J, Bruzzone L, Buiatti M (2011) Adjust: an automatic EEG artifact detector based on the joint use of spatial and temporal features. Psychophysiology 48(2):229-240

24. Hilz MJ, Stemper B, Kolodny EH (2000) Lower limb cold exposure induces pain and prolonged small fiber dysfunction in Fabry patients. PAIN 84(2-3):361-365

25. Le Pera D, Svensson P, Valeriani M, Watanabe I, Arendt-Nielsen L, Chen ACN (2000) Long-lasting effect evoked by tonic muscle pain on parietal EEG activity in humans. Clin Neurophysiol 111(12):2130-2137

26. Vuckovic A, Gallardo VJF, Jarjees M, Fraser M, Purcell M (2018) Prediction of central neuropathic pain in spinal cord injury based on EEG classifier. Clin Neurophysiol 129(8):1605-1617 
27. Jensen MP, Hakimian S, Sherlin LH, Fregni F (2008) New insights into neuromodulatory approaches for the treatment of pain. $J$ Pain 9(3):193-199

28. Vogel W, Broverman DM (1964) Relationship between EEG and test intelligence: a critical review. Psychol Bull 62(2):132

29. Cong F, Kalyakin I, Ristaniemi T, Lyytinen H (2008) Drawback of ICA procedure on EEG: polarity indeterminacy at local optimization. In: 14th Nordic-baltic conference on biomedical engineering and medical physics. Springer, Berlin, pp 202-205
Publisher's Note Springer Nature remains neutral with regard to jurisdictional claims in published maps and institutional affiliations. 